\begin{tabular}{lllllllllllllllllllllllllllllllll}
\hline$R$ & $E$ & $V$ & I & S & T & A & D & E & E & S & T & U & D & I & O & S & I & N & T & E & R & N & A & C & I & O & N & A & L & E & S
\end{tabular}

\title{
Visiones globales y opciones transnacionales en la agenda exterior chilena con Estados Unidos. Aproximación a un estudio de caso*
}

\author{
José A. Morandé Lavín
}

\begin{abstract}
A partir de un estudio de caso, este ensayo pretende abordar las visiones y preferencias cosmopolitas de elites locales formadas en centros académicos norteamericanos en relación a la política exterior de Chile con Estados Unidos. Más específicamente y sobre la base de la experiencia de intercambio académico del Programa Fulbright en nuestro país, se intenta discutir conceptual y analíticamente algunos hallazgos preliminares que dan cuenta de cómo la globalización y creciente interdependencia de la sociedad civil internacional estarían incidiendo en un interés de las elites chilenas por influenciar en las políticas públicas de Chile, en particular en lo que se refiere a su inserción internacional. Del mismo modo, los principios y valores transnacionales que orientan dichos grupos de interés, se sustentarían en los vínculos e interacciones educacionales facilitados por entidades de cooperación cultural como la Comisión Fulbright. De esta forma, la influencia ejercida por la comunidad académica y científica estadounidense con sus pares chilenos habría sido funcional para una aproximación más afín en las visiones y opciones culturales entre ambos países.
\end{abstract}

* Esta publicación forma parte de un estudio sobre visiones de las elites chilenas en política exterior con Estados Unidos, apoyado inicialmente por el Proyecto de Enlace 2003 ENL 03/07 de la Vicerrectoría de Investigación y Desarrollo de la Universidad de Chile y actualmente en ejecución en el marco del Proyecto de Investigación $N^{\circ} 1040561$, financiado por Fondecyt-Chile. 
INTRODUCCIÓN: DIPLOMACIA CULTURAL Y LIDERAZGO GLOBAL NORTEAMERICANO

EN LA INSERCIÓN INTERNACIONAL DE CHILE

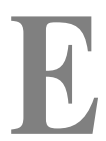

1 dinamismo del sistema internacional contemporáneo y su transformación así como las tendencias globales de la política mundial han tenido repercusiones importantes en las visiones y opciones de inserción internacional de Chile los últimos cincuenta años. En efecto, en un período de más de medio siglo, su agenda exterior ha estado configurada, bajo el liderazgo de Estados Unidos, desde el contenido político-estratégico de la Guerra Fría hasta la reciente globalización de la economía y la sociedad mundial ${ }^{1}$.

No obstante la tradicional inserción internacional chilena y su identificación con los principios universales y los valores cosmopolitas del mundo occidental, los sucesivos gobiernos han procurado diseñar y aplicar una política exterior y de Estado orientada a incrementar y consolidar la autonomía y la identidad nacionales a partir de estrategias de desarrollo que permitan alcanzar etapas superiores de crecimiento económico y bienestar social. Es más, este aspecto de la política exterior chilena es parte de una larga tradición sustentada en su identidad política y en el peso de sus instituciones democráticas. Asimismo, a lo largo de la historia la imagen internacional de Chile se ha acompañado del predominio de una cultura política interna que ha valorado la participación en el mundo occidental, con especial énfasis en su adhesión a sus valores políticos y a las visiones sobre el desarrollo económico y social que éste representa. En consecuencia, la evolución del entorno internacional tiende a la interacción entre variables sistémicas e internas y constituye un apropiado esquema de análisis para comprender las visiones y opciones transnacionales sobre la agenda exterior de actores y representantes de la sociedad chilena.

\section{Una de las manifestaciones de la nueva realidad social es la internacionalización creciente de las elites locales.}

Desde el fin de la Guerra Fría y la rearticulación de la política mundial y regional en torno a la expansión de la democracia y la competencia de la economía liberal, la agenda y la conducta exterior de Chile han estado insertas en una estrategia más internacional. Asimismo, en el plano interno, la política externa ha estado coincidentemente orientada e influenciada por los mismos valores que sustentan los amplios y profundos cambios políticos y económicos que ha experimentado la sociedad civil chilena en décadas

${ }^{1}$ Sobre los rasgos de la inserción internacional de Chile en las últimas décadas, véase: Morandé, José A. "Chile: The Invisible Hand and Contemporary Foreign Policy", en Frank O. Mora y Jeanne A. K. Hey, eds., Latin American and Caribbean Foreign Policy (Lanham, MD: Rowmann \& Littlefield Publishers, Inc., 2003), pp. 243-264. 
recientes, entendiendo por sociedad civil al conjunto de organizaciones no estatales que cubre el espacio político y social de la comunidad nacional e internacional.

Una de las manifestaciones de esta nueva realidad social es la internacionalización creciente de las elites locales en torno a los valores transnacionales que representa la globalización actual de la política mundial. En otras palabras, las ideas y actitudes de estas elites reflejan, en parte, una comunidad internacional cada vez más global e interdependiente. Este es el caso de muchas personas y grupos de intereses en Chile que se han formado en los centros culturales y tecnológicos más avanzados del mundo y que actualmente tienen visiones y conductas más cosmopolitas en la proyección internacional del país. Interesa examinar entonces, a la luz del estudio de las recientes transformaciones internacionales y de la política exterior de Chile en las últimas décadas, las visiones y la conducta internacional de las elites locales.

\section{Mediante becas individuales el programa cultural apuntóa internacionalizar la educación estadounidense.}

La inserción chilena en la esfera de influencia política, económica y cultural de los Estados Unidos sumada al indiscutido liderazgo de este país en el plano mundial y hemisférico, son factores gravitantes y determinantes para comprender el grado de mundialización de las elites nacionales. De ahí que una manera de ilustrar este fenómeno es auscultar el nivel de transnacionalización de dichos grupos, a partir de sus visiones, actitudes y adhesiones a los valores e ideas que sustenta y representa la sociedad del país rector del planeta.

El presente trabajo forma parte de una investigación en curso que da cuenta de las visiones y preferencias transnacionales de las elites chilenas en opciones sobre la política exterior respecto de Estados Unidos. Para ello, se anticipan algunos resultados y conclusiones preliminares de una encuesta a personas que residieron y se formaron en los Estados Unidos, para lo cual se considera como estudio de caso una institución de intercambio cultural y científico como es el Programa Fulbright en Chile. En otras palabras, se trata de observar percepciones y opciones internacionales de grupos profesionales y académicos chilenos que fueron beneficiados con becas de perfeccionamiento gracias al patrocinio y al apoyo de un acuerdo de intercambio cultural como el seleccionado para esta indagación. Así, desde su instalación en Chile, en virtud de un Acuerdo entre los gobiernos de ambos países suscrito el 31 de marzo de 1955 , este convenio ha beneficiado con becas en centros académicos de mayor prestigio en Estados Unidos a más de 1.400 profesionales chilenos; por su parte más de 700 especialistas estadounidenses han tenido la oportunidad de venir a Chile ${ }^{2}$.

\footnotetext{
${ }^{2}$ Ministerio de Relaciones Exteriores de Chile, Dirección de Prensa y Difusión. http://www.minrel.cl/ prensa/comunicados 2003/19-06-03.htm
} 


\section{La creciente interdependencia de la sociedad civil en el sistema internacional contribuiría a una mayor presencia de las elites chilenas en la agenda exterior del país.}

Por lo tanto, la experiencia de intercambio académico-profesional constituye un estudio de caso para examinar, entre otras cosas, el papel desempeñado por el Programa Fulbright en Chile. Particularmente interesa evaluar esta experiencia como instrumento de política exterior de los Estados Unidos en los campos de la educación y de la cooperación e integración culturales entre ambos países. En efecto, desde la década de 1950 ha operado en Chile el convenio con la Fundación Fulbright, apoyado inicialmente con fondos del gobierno estadounidense destinados a gastos no militares, y con el propósito de contribuir a la diplomacia pública de Estados Unidos y a una mejor comprensión del mundo por parte de sus ciudadanos. En febrero de 1997, los gobiernos de Estados Unidos y Chile firmaron un nuevo Acuerdo en materia de cooperación educacional, para lo cual se creó una Comisión bilateral para el intercambio educativo -de carácter binacional y financiada con fondos de ambas partescon el fin de continuar el trabajo realizado hasta ese entonces por la Comisión para el Intercambio Educacional entre Chile y los Estados Unidos de América, creada en el mes de marzo del año 1955. Originalmente, los objetivos del programa cultural destacaban el apoyo a la internacionalización de la educación estadounidense, ayudando a desarrollar a través de becas individuales, la "cultura cívica" norteamericana, considerada como uno de los componentes necesarios para el funcionamiento de una sociedad democrática ${ }^{3}$.

Debido a lo anterior, la actividad de intercambio académico desarrollada por la Comisión en nuestro país, es una excelente oportunidad para explicar las percepciones y conductas de las elites locales formadas y especializadas en centros académicos estadounidenses acerca de la inserción internacional de Chile y su eventual influencia en las opciones de política exterior con los Estados Unidos. Este estudio establece como hipótesis de trabajo que la creciente globalización e interdependencia de la sociedad civil en el sistema internacional contribuirían a un mayor interés, presencia e influencia de las elites chilenas en la definición de la agenda exterior del país, en particular con relación a Estados Unidos. Del mismo modo, los principios y valores transnacionales que estarían asumiendo y expresando estos grupos de intereses, se originarían en

\footnotetext{
${ }^{3}$ Acerca del origen y trayectoria del Programa Fulbright en el mundo, véase a Sussman, Leonard R., The Culture of Freedom. The Small World of Fulbrights Scholars, Lanham, MD, Rowmann \& Littlefield Publishers, Inc., 1992, pp. 37-38. En el caso de la transformación reciente de la Comisión en Chile, véase la promulgación del "Acuerdo con los Estados Unidos de América en materia de Cooperación Educativa", Decreto del Ministerio de Relaciones Exteriores de Chile, n 2.040 del 23 de noviembre de 1998.
} 
vínculos e interacciones educacionales facilitados por entidades binacionales de cooperación cultural. Tal es el caso de la influencia que a través de estos medios ha ejercido en las elites locales la comunidad científica y académica norteamericana, cuya contrapartida, es que dichos sectores de la sociedad nacional apunten a influir en pro de una aproximación política, económica y cultural de Chile más marcada y coincidente con los Estados Unidos.

\section{AsPectos CONCEPTUALES DEL}

ANÁLISIS DE LA AGENDA EXTERIOR

Y DE LA PROYECCIÓN INTERNACIONAL DE LAS ELITES CHILENAS

Entre los procesos más destacados que experimenta la política mundial contemporánea se destaca la expansión de la sociedad civil internacional y la creciente preocupación y participación de actores no estatales y grupos de intereses en los problemas y materias de la agenda internacional. Esta tendencia participativa y global, junto con reconocer la influencia a veces determinante que ejercen tales agentes en las políticas públicas, también admite la transmisión de creencias y valores, los que pueden convertirse en factores decisivos para legitimar políticas de
Estado en relación con las demandas de la sociedad civil ${ }^{4}$.

Del mismo modo, el hecho de que muchas materias que preocupan a la humanidad sean de carácter transnacional la creciente vinculación e interdependencia de los factores y externos de la política internacional contribuyen a cuestionar -por parte de las elites nacionales- el papel y preeminencia absoluta de los valores clásicos de la soberanía de los Estados en el tratamiento de algunos aspectos de alcance más amplio y reformular sus visiones tradicionales de apego a lo estrictamente nacional ${ }^{5}$. A este respecto, la discusión conceptual reciente acerca de la confrontación de los aspectos nacionales/ cosmopolitas de algunas comunidades de la sociedad internacional, permite identificar y explicar conductas de elites transnacionales como las que interesan en este estudio.

\section{Aumenta la participación de actores no estatales en los problemas de la agenda internacional.}

Los primeros, vale decir los sectores o grupos nacionalistas, se identifican fuertemente con su nación-Estado y tienen poca confianza en las instituciones multilaterales e internacionales. Además, fa-

\footnotetext{
${ }^{4}$ Pomerleau, Claude; Morandé, José A. "Dimensiones conceptuales y ético-religiosas de la agenda internacional del medio ambiente para el siglo XXI", Estudios Internacionales, n ${ }^{\circ} 127-128$, septiembrediciembre de 1999, pp. 104-105.

${ }^{5}$ Rosenau, James N. "Preteorías y teorías sobre política exterior", en J. A. Vásquez, (comp.), Relaciones internacionales. El pensamiento de los clásicos, México, D.F., Editorial Limusa, 1994, pp. 206-219.
} 
vorecen políticas de proteccionismo económico por encima de la libertad comercial de bienes y servicios. Las elites cosmopolitas, por el contrario, se identifican más ampliamente con su continente o con el mundo en su conjunto y en general se sienten más cómodos viviendo y trabajando en diferentes países. Del mismo modo, están más habituados a los viajes más allá de las fronteras nacionales, dominan idiomas, y están conectados a redes internacionales a través de comunicaciones mundiales ${ }^{6}$.

\section{La discusión conceptual sobre los aspectos nacionales/cosmopolitas en algunas comunidades permite identificar conductas de las elites}

\section{transnacionales.}

Sin embargo, los procesos políticos que se generan a partir de estos nuevos imperativos de la realidad internacional reclaman no sólo la participación de actores que operan en el ámbito externo al Estado, sino también la incorporación creciente de agentes gubernamentales en esta misma dinámica. Muchos de estos personeros públicos han asimilado valores transnacionales desde el momento en que se formaron centros académicos y profesionales internacionales altamente calificados, particularmente de Estados Unidos y de Europa. Así, visiones neo-rrealistas de las relaciones internacionales, sin perjuicio de reiterar el papel gravitante del Estado en la política mundial, contribuyen a resaltar esta interacción transnacional entre agentes privados y gubernamentales ${ }^{7}$. En otros términos, las conductas y resultados transnacionales bajo esta visión son evaluadas en función de las capacidades e influencias que ejercen distintos actores, públicos y privados. Es más, los Estados, principalmente los más poderosos, tienen gran influencia en las estructuras institucionales y en las reglas y normas que adoptan actores transnacionales. En consecuencia, es necesario tanto identificar estos agentes a través de organizaciones locales o de redes de interacciones transnacionales en las cuales participan, y comprender las modalidades de expresión y acción de dichos grupos a fin de influir en la formulación de las políticas públicas.

En la misma línea de la influencia de Estados poderosos en las visiones que tienen las elites transnacionales, cabe mencionar el aspecto cultural de la política internacional. Particularmente relevante es el significado y alcance que han adquirido

${ }^{6}$ Norris, Pipa. "Global Governance and Cosmopolitan Citizens", en Nye Jr. Joseph S. y John D. Donahue, (eds.), Governance in a Globalizing World, Washington, D.C., Brookings Institution Press, 2000, pp. 158-159.

${ }^{7}$ La perspectiva "estatista" de Krasner identifica al Estado como "el conjunto de actores e instituciones que adoptan las decisiones públicas centralmente al interior de la organización". A este respecto, véase Krasner Stephen D., " Power politics, institutions, and trasnational relationes”, en Thomas RisseKappen, (ed.), Bringing Transnational Relations Back In. Non-State Actors, Domestic Structures and International Institutions, Cambridge, Cambridge University Press, 1999, p. 258. 
los conceptos de "poder duro" ("hard power”) y "poder blando"(“soft power”) acuñados por Joseph S. Nye cuando explica los principios e instrumentos tradicionales de la política exterior de Estados Unidos, y que cobran fuerza en el mundo de la post Guerra Fría. El "poder duro" representa el poder tradicional que otorgan la coerción y la fuerza militar; en cambio, la ideología liberal justifica el "poder suave", como factor de adquisición de prestigio, para lo cual sus principios pueden ser una fuente de poder, esto es, intangibles tales como "la atracción cultural, los valores políticos y las fortalezas sociales que otros admiran"8. En otras palabras, se trata de atraer a otros por la legitimidad de las políticas estadounidenses y los valores que las sustentan. Del mismo modo, Mark Falcoff destaca que durante un largo período, como lo fue la Guerra Fría la política de Estados Unidos hacia América Latina y en particular hacia Chile, combinó instrumentos de políticas "duras" y "suaves". Entre los últimos destaca al programa de becas Fulbright, que sobre la base de una cooperación cultural y educacional no sólo contribuyó a divulgar valores y principios universales de interés mutuo, sino también contrarres- tó así los efectos negativos de la intervención unilateral estadounidense en la región?.

\section{Las visiones neorrealistas de las relaciones internacionales contribuyen a resaltar la interacción transnacional entre agentes privados $y$ gubernamentales.}

No obstante los aportes funcionales de la perspectiva estatista a los estudios de relaciones internacionales para analizar la interacción entre instituciones y actores estatales y privados en el mundo global actual, el marco teórico más apropiado para abordar los vínculos internos e internacionales de las elites chilenas y su grado de influencia en la política exterior es el que se identifica tempranamente como el paradigma Transnacional y de la Interdependencia Compleja ${ }^{10}$. Este enfoque parte de la base de que en el sistema internacional contemporáneo existen relaciones transnacionales como contactos, coaliciones e interacciones que trascienden los límites del Estado y que no están necesariamente bajo el control

${ }^{8}$ Sobre estos conceptos y su influencia en la política norteamericana véase: Nye, Jr., Joseph S., "The Decline of America's Soft Power: Why Washington Should Worry" en Foreign Affairs, vol. 83, n 3 , May/June 2004, pp. 16-18. Ver también Nye Jr., Joseph S., en Bruce W. Jentleson, American Foreign Policy: The Dynamics of Choice in the 21 st Century, Nueva York, W.W. Norton \& Company, 2000, pp. 400-402.

${ }^{9}$ Falcoff, Mark. "A Look at Latin America”, en Nicholas X. Rizopoulos, (ed.), Sea-Changes: American Foreign Policy in a World Transformed, Nueva York, Council on Foreign Relations Press, 1990, p. 73.

${ }^{10}$ Acerca de esta pespectiva teórica, véase el libro clásico de Robert O. Keohane y Joseph S. Nye Jr., Transnational Relations and World Politics, Cambridge, Mass. Harvard University Press, 1971. 
de los mecanismos centrales de los gobiernos. Es más, las interacciones globales ocurren en varios campos, destacándose en primer lugar el de las comunicaciones, donde el movimiento de información consistente en la transmisión de ideas, creencias y doctrinas constituye un sustrato importante de muchas de las actividades internacionales del presente. $\mathrm{La}$ actualización de este marco teórico se encuentra en la propia reflexión de Keohane y Nye, cuando se refieren a los procesos actuales de globalización y gobernabilidad mundial. Distinguiendo analíticamente entre globalización y globalismo, entendido este último como "un estado del mundo que involucra redes de interdependencia a distancias multicontinentales" "11, ambos autores profundizan acerca de las redes de interdependencia contemporáneas, que actualmente se refieren a conexiones o relaciones múltiples a escala global. La globalización, por su parte, encierra procesos en marcha de creación de globalismos de diferente naturaleza. Entre los aspectos destacan el globalismo social y cultural, que encierra movimientos de ideas, información, imágenes y, por cierto, movimiento de personas que comparten sus mismas creencias y valores.

En el marco anterior es necesario explorar los efectos que tienen las interacciones del globalismo cultural y social en la conciencia de los individuos y en sus actitudes hacia la cultura, la política y la identidad personal y social. Para nuestro estudio de caso, el reconocimiento de esta dimensión y atendiendo a que actualmente Estados Unidos ha consolidado y mantenido una posición preponderante como el mayor generador de una cultura de consumo en el mundo, permite sostener la difusión y el impacto de sus ideas y valores a nivel global y local. Es más, la expansión de elementos de la ideología estadounidense y de sus estilos de vida provee de diferentes audiencias en la sociedad internacional, con un sentimiento de conexión simultáneo a los Estados Unidos ${ }^{12}$.

\section{El Programa Fulbright contribuyó a contrarrestar los efectos negativos de la intervención unilateral estadounidense en la región.}

El referente conceptual transnacional y de la interdependencia compleja, desarrollado a través de diferentes modelos, permite explicar variadas relaciones entre actores transnacionales y gobiernos, al igual que a las relaciones interestatales. Una versión más funcional de las relaciones transnacionales se encuentra en la obra de Thomas RisseKappen ${ }^{13}$ quien incorpora los conceptos de relaciones "trans-sociales" y "transgubernamentales", referidas en general

${ }^{11}$ Nye Jr., Joseph S.; Keohane, Robert O. "Introduction”, en Joseph S. Nye y John D. Donahue, (eds.), op. cit., pp. 2-5.

${ }^{12}$ Rosendorf Neal M. "Social and Cultural Globalization: Concepts, History, and America's Role", en Joseph S. Nye y John D. Donahue, (eds.), op. cit., p. 128. 
a interacciones períodicas a través de las fronteras nacionales, donde al menos un actor es un agente no estatal o no actúa en favor de un gobierno nacional o de una organización intergubernamental.

\section{Deben investigarse los efectos de las interacciones del globalismo cultural y social en la conciencia de los individuos.}

Por otra parte, la realidad transnacional de la sociedad internacional contemporánea facilita la interacción de grupos de intereses y la incursión de éstos en el escenario internacional. La explicación teórica de este renovado papel de actores transnacionales en el contexto de la política mundial de nuestros días ha cobrado también importancia en los estudios acerca de la gobernabilidad global y la sociedad civil internacional. De esta manera, la sociedad global de hoy provee un escenario en que los actores pueden idear y organizar los recursos materiales para transformar la matriz institucional en que se insertan sus expresiones y conductas ${ }^{14}$. En otras palabras, los esfuerzos de muchas organizaciones no gubernamentales pueden persuadir a los gobiernos nacionales que adoptan ciertas recomendaciones de política pública en materia de ne- gociaciones internacionales o bien, trabajar fuera del sistema estatal para crear principios y valores junto con desarrollar normas que influyan en el pensamiento y la conducta de las personas y de las colectividades en el mundo. Así, la incorporación de interacciones transnacionales, transgubernamentales e interestatales en el contexto de una sociedad civil global e interdependiente ofrece una perspectiva amplia de análisis para examinar tanto las percepciones como el comportamiento internacional de grupos de intereses o elites transnacionales.

Estas tendencias y procesos internacionales se pueden apreciar en algunas expresiones tales como la promoción de valores compartidos, la concertación de políticas específicas entre pares y acciones conjuntas a nivel transfronterizo. En el caso chileno, las elites locales habrían asumido un papel activo en la inserción internacional de Chile a partir de las transformaciones recientes de la política mundial, del sistema político chileno como también de su estrategia de desarrollo de apertura externa. En otras palabras, las premisas neoliberales de la economía política internacional son funcionales a la expansión de la sociedad civil chilena y a la influencia creciente de sus grupos de intereses en la política exterior. Más específicamente, mientras menor sea la presen-

${ }^{13}$ Véase Risse-Kappen Thomas, “Bringing Transnational Relations Back In: Introduction”, en Thomas Risse-Kappen, (ed.), Bringing Transnational Relations Back In. Non-State Actors, Domestic Structures and International Institutions, Cambridge, Cambridge University Press, 1999, pp. 7-9.

${ }^{14}$ Wapner Paul, "Governance in Global Civil Society", en Oran R. Young, (ed.), Global Governance. Drawing Insights from the Environmental Experience, Cambridge, Mass, The MIT Press, 1997, pp. 6584. 
cia del Estado en las decisiones públicas, mayor será el desarrollo de actores no gubernamentales y creciente la influencia y alcance de la participación de las elites locales en la proyección internacional del país.

\section{Las premisas neoliberales de la economía política internacional son funcionales a la expansión de la sociedad civil chilena.}

Desde la perspectiva analítica de la política exterior y en función de los objetivos de este estudio, los referentes teóricos seleccionados apuntan a los que se centran en perspectivas o visiones del sistema internacional y en las orientaciones y procesos de decisión de los Estados. Las variables sistémicas que intervienen en la política exterior se refieren a todas aquellas acciones que tienen lugar fuera del ámbito nacional, tanto realidades geográficas como desafíos ideológicos, que condicionan o influyen de alguna manera en los decisores locales y en los responsables de la política internacional de un país. En cuanto a las visiones internas que influyen en política exterior, interesa destacar las variables gubernamentales, esto es, las orientaciones estratégicas y las estructuras de gobierno que facilitan o restringen las opciones de política exterior, así como las variables no gubernamentales y culturales que influyen en las visiones de la sociedad civil y que apuntan a las principales tendencias en materia de valores y aspiraciones externas de una nación ${ }^{15}$. En general, los procesos políticos que acompañan a las decisiones de política exterior se configuran por estas fuerzas sociales y culturales. Ambos aspectos, entonces, son gravitantes desde el momento en que la cultura representa condiciones, predisposiciones y actitudes generalizadas de una población mientras que la sociedad, es una manifestación particular de la cultura ${ }^{16}$.

A nivel sistémico de la política exterior, los modelos de la sociedad global e interdependencia compleja ya mencionados, contemplan entre sus supuestos la existencia de una sociedad global con una extensa y variada agenda de temas en el marco de una compleja interdependencia estructural, donde los Estados naciones han llegado a ser altamente permeables a las presiones de una amplia gama de actores no estatales y de organizaciones internacionales de distinta índole. Los procesos centrales del sistema internacional contemplan efectos agregados de decisiones que provienen de actores nacionales $y$ no nacionales en un universo de demandas correspondiente a una agenda internacional en que figuran de manera creciente amenazas y oportunidades surgidas de las necesidades actuales de la humanidad. Asimismo, los modelos de la sociedad global e interdependencia compleja son más sensibles a incorporar en su agen-

${ }^{15}$ Rosenau James N., op. cit., p. 208.

${ }^{16}$ Fry, Earl H.; Taylor, Stan A.; Wood, Robert S. America the Vincible: U.S. Foreign Policy for the Twenty-First Century, Englewood Cliffs, N. J., Prentice-Hall, Inc., 1989, pp. 104-105. 
da aspectos relacionados con políticas comerciales, migraciones, salud, medio ambiente y otros temas, que no son vistos con prioridad por las perspectivas tradicionales del Estado. Por lo tanto, este paradigma internacional sitúa el tema de las visiones y la conducta de las elites en política exterior en el mundo de hoy como un fenómeno que trasciende los límites tradicionales de los Estados-naciones y se explica en función de una lógica global e interdependiente ${ }^{17}$.

\section{Los procesos políticos que acompañan las decisiones de política exterior se configuran por fuerzas sociales y culturales.}

En cuanto a las variables internas de la política exterior y, en particular a los efectos de nuestro estudio de caso, es importante consignar en primer término los modelos grupales que operan en los procesos decisionales a partir de la interacción en torno a la confrontación de creencias y valores entre pequeños grupos de elites internos y transnacionales. A este respecto, el concepto de "Domestic-Foreign Frontier" de Rosenau constituye un valioso marco de análisis para explicar estas interacciones cuando se refiere a los desafíos de la gobernabilidad mundial sobre la base de la expansión de las fronteras de los Estados-naciones, desde lo interno a lo internacional. Es decir, el proceso contemporáneo de confrontación entre lo global y lo local, junto con originar una permeabilidad creciente de los Estados a las influencias transnacionales, ha generado también un espacio ("frontera") en el cual interactúan diversos agentes en una variedad de temas y a través de los cuales se vinculan los aspectos locales, nacionales e internacionales de la política mundial ${ }^{18}$. En consecuencia, la conducta externa respecto de las percepciones y visiones de grupos sociales y particularmente de sus elites, es determinante para comprender y evaluar los procesos de política internacional. Estudios más recientes sobre el papel de actores no gubernamentales en la política exterior se refieren a las experiencias de servicio y aprendizaje del mundo intelectual en este ámbito, es decir la puesta a prueba de la teoría por especialistas, a partir de situaciones y casos concretos ${ }^{19}$.

Asimismo, y desde una perspectiva latinoamericana, los enfoques sobre estrategias de desarrollo también constituyen una valiosa contribución a la conducta de las elites y su influencia en la actual inser-

${ }^{17}$ Holsti Ole R.. "Models of International Relations and Foreign Policy", en G. John Ikenberry, (ed.), American Foreign Policy. Theoretical Essays, Harper Collins: College Publishers, 1995, pp. 72-74.

${ }^{18}$ Rosenau, James N., Along the Domestic-Foreign Frontier. Exploring Governance in a Turbulent World, Cambridge, Cambridge University Press, 1997, pp. 3-11.

${ }^{19}$ Jentleson Bruce W., "In Pursuit of Praxis: Applying International Relations Theory to Foreign Policy-Making", en Miroslav Nincic y Joseph Lepgold, (eds.), Being Useful. Policy Relevance and International Relations Theory, Ann Arbor, The University of Michigan Press, 2000, pp. 129-149. 
ción internacional de Chile ${ }^{20}$. En particular, esta perspectiva analítica se concentra en el papel fundamental de las burocracias como escenarios de competencia entre tecnócratas que buscan usar sus contactos con comunidades extranacionales para fines de política interna. Del mismo modo, en la elaboración de una estrategia de desarrollo que propenda al crecimiento y la sustentación económica, los grupos que constituyen la elite política se preocuparán de cómo los actores económicos no nacionales, como las empresas multinacionales o los organismos internacionales de crédito, pueden ser inducidas a contribuir a los objetivos nacionales definidos por las elites nacionales. En otras palabras y conforme a esta visión de la política exterior, las opciones de desarrollo se relacionan con la necesidad de permanencia de las elites políticas y con la forma en que éstas manipulan las condiciones internacionales para el logro de los objetivos de dicha estrategia.

\section{La conducta de las elites en política exterior es un fenómeno que trasciende los límites tradicionales de los Estados-naciones.}

Por último, en el ámbito de las decisiones de política exterior, el modelo de dinámica de grupos $^{21}$ aporta a la discusión teórica del tema bajo estudio, desde el momento en que este modelo define la toma de decisiones como el resultado de la interacción grupal, de acuerdo con el cual la mayoría de las decisiones son adoptadas por grupos de elite en un proceso dinámico. De esta manera el papel de las elites en política exterior se puede explicar y evaluar considerando simultáneamente tanto el nivel de análisis del sistema internacional como el de los Estados, en particular en lo que se refiere a la forma en que las orientaciones y procesos de formulación de la política exterior se relacionan con las percepciones y el comportamiento de sus elites.

\section{EVIDENCIAS DE UNA PARTICIPACIÓN Y APROXIMACIÓN TRANSNACIONALES CON \\ ESTADOS UNIDOS}

De acuerdo con la hipótesis de trabajo adoptada para el estudio de caso seleccionado y sobre la base de resultados preliminares de la encuesta realizada a representantes de elites chilenas formadas en Estados Unidos, por conducto del Programa Fulbright, pueden apreciarse las siguientes frecuencias en las visiones y opciones respecto del grado de cosmopolitismo de las elites y la imagen de éstas acerca de Estados Unidos y su papel en el escenario internacional, en particular en la relación bilateral con Chile ${ }^{22}$.

${ }^{20}$ Coleman Kenneth M. y Luis Quiros-Varela, "Determinants of Latin American Foreign Policies: Bureaucratic Organizations and Development Strategies", en Elizabeth G. Ferris y Jennie K. Lincoln, (edits.), Latin American Foreign Policies, Boulder, Co: Westview Press, 1982, pp. 39-59.

${ }^{21}$ Holsti Ole R., op. cit., pp. 79-80.

${ }^{22}$ La información empírica sobre las visiones de las elites chilenas formadas en Estados Unidos se 
La totalidad de los entrevistados afirman estar de acuerdo en que la globalización exige una creciente especialización y la participación de expertos y profesionales en las políticas públicas. Esta expresión refleja una inclinación tecnocrática, por cuanto favorece los requerimientos de especialistas altamente calificados para el desempeño de funciones del Estado, complementándose con una apreciación mayoritaria de apoyo -más del $80 \%$ en cuanto al imperativo de que la elaboración de la política exterior de Chile responda a criterios técnicos y sea formulada por profesionales.

Asimismo, la apreciación de los entrevistados respecto de la evaluación técnica de los logros chilenos en materia de política exterior es casi unánime. En efecto, el $97 \%$ de la muestra señala que los acuerdos de libre comercio suscritos por Chile son el resultado del trabajo de profesionales altamente calificados. Desde el punto de vista de la relación bilateral con Estados Unidos, se observa una percepción ampliamente optimista, ya que hay unanimidad en cuanto a que el Tratado de Libre Comercio suscrito entre ambos países no sólo incrementará las relaciones bilaterales sino que facilitará una mayor cooperación y entendimiento mutuo.

Pese a la evaluación positiva de las elites chilenas acerca de los vínculos bilaterales de nuestro país con Estados Unidos, al mismo tiempo es posible identificar altos índices de desaprobación de la política exterior estadounidense a nivel mundial y regional. En efecto, $62 \%$ de los encuestados no cree que la intervención en Irak haya sido por motivos de lucha contra el terrorismo internacional. Este desacuerdo respecto de la intervención estadounidense en el exterior aumenta tratándose de América Latina. Así, frente a la pregunta de si Estados Unidos debiera intervenir en Colombia para controlar el narcotráfico y el terrorismo regional y mundial, la desaprobación aumenta al 92\% de los entrevistados. Lo mismo ocurre con el rechazo al mantenimiento de las sanciones económicas y el embargo comercial que el país del Norte mantiene con Cuba, ya que casi $90 \%$ de los consultados están de acuerdo en levantar las prohibiciones.

\section{La conducta externa respecto de las percepciones de las elites es determinante para comprender $y$ evaluar los procesos de política internacional.}

Sin embargo, la autopercepción y la autoconfianza de la elites nacionales respecto de Chile y de su relación positiva y favorable a nivel latinoamericano y hemisférico con Estados Unidos es elocuente. A pesar de desaprobar la intervención norteamericana en la región, las elites muestran en parte el papel diferenciador de Chile en el contexto regional. De este modo, frente a la consulta de si Estados 
Unidos necesita a Chile como socio confiable y creíble en Latinoamérica, un $86 \%$ aprueba la idea de asociación y participación bilateral.

\section{Las opciones de desarrollo se relacionan con la necesidad de permanencia de las elites políticas}

Desde el punto de vista de la identificación de las elites chilenas formadas en Estados Unidos, particularmente en el aspecto nacional /cosmopolita, sus respuestas son ambivalentes en el sentido de que pese a reconocer y adherir a principios y valores transnacionales, no se advierte un abandono, ni renuncia a los valores nacionales. Puede así advertirse una aceptación de los mecanismos y medios que entrega la globalización de la sociedad internacional. Al respecto, el reconocimiento de los entrevistados de que los medios de comunicación contribuyen a conectarlos y acercarlos al mundo es significativo, ya que su aprobación representa más del $92 \%$. Asimismo, la elevada importancia que atribuyen las elites al dominio del idioma inglés como requisito para funcionar e interactuar en la vida actual es calificada positivamente por cerca de un $98 \%$ de los entrevistados. No obstante, la identidad de las elites con lo nacional también es asumida por éstas conforme a los compromisos con la realidad local, con su historia y con grados de aceptación y satisfacción sugerentes en cuanto al desarrollo y a la proyección de país.

Lo anterior se comprueba en primer lugar cuando se rechaza la opción por lo global frente a los imperativos nacionales. Del mismo modo y de acuerdo con la identificación de las elites locales con lo nacional, existe una aprobación general acerca del funcionamiento de la democracia chilena, de alrededor del $83 \%$ de conformidad. Esta cifra puede interpretarse como un grado alto de satisfacción frente a los procesos políticos y sociales que ha experimentado el país en los últimos años. Por último, en esta misma categoría de compromiso e identificación con lo estrictamente nacional de las elites locales, cabe resaltar la respuesta categórica en relación con lo que ha sido la visión tradicional de la política exterior chilena con Bolivia. En efecto, ante la pregunta de si Estados Unidos debiera ayudar a una respuesta a las demandas marítimas de dicho país con Chile, el rechazo de los entrevistados es unánime.

Por otra parte, la influencia cultural que la política estadounidense habría ejercido en las elites locales se puede apreciar, en parte, en las percepciones y opiniones de estos grupos acerca de las instituciones estadounidenses. En general, existe una receptividad y evaluación positivas del nivel de excelencia de los centros académicos y universitarios de Estados Unidos. Esto es particularmente efectivo en cuanto cerca de un $70 \%$ de los encuestados aprueba la afirmación de que la mejor formación de los profesionales y especialistas chilenos se alcanza en dichos centros de especialización. Esta tendencia indicaría preliminarmente una autoapreciación educacional alta de las elites chilenas que se han formado en el exterior, asumiendo y corroborando de algún modo 
una evaluación favorable del nivel de especialización y excelencia de los centros académicos y profesionales en que tuvieron la oportunidad de estudiar y trabajar en Estados Unidos.

\section{Las elites asumen la identidad con lo nacional conforme a los compromisos con la realidad local.}

En síntesis, un análisis simple y parcial de los resultados encontrados en la encuesta sobre nuestro grupo de estudio, nos permite concluir preliminarmente que hay una elite local con rasgos evidentes de transnacionalización y cosmopolitismo. Pese a que este sector asume los desafíos y oportunidades que ofrece la globalización de la sociedad civil contemporánea, se proyecta internacionalmente sin renunciar a valores y actitudes de su propia identidad nacional. Al mismo tiempo, las conclusiones preliminares de la encuesta verifican parcialmente la hipótesis de trabajo de este estudio, en el sentido de que habría cierta preferencia y aproximación especiales de estas elites respecto de Estados Unidos, tanto en las opciones políticas -aunque con reservas en el ámbito global y hemisférico- como en los aspectos económicos y culturales.

\section{CONSIDERACIONES FINALES}

A comienzos del siglo XXI, Chile se encuentra inserto internacionalmente conforme a los principios políticos democráticos y las orientaciones económicas libe- rales que predominan tanto en el ámbito de la política mundial como interna. Los valores transnacionales de la sociedad civil globalizada, sustentados y promovidos bajo el liderazgo indiscutido de Estados Unidos han tenido un impacto e influencia gravitantes en la formación y en las visiones de las elites chilenas respecto de las políticas públicas nacionales.

Por lo que respecta a los actores seleccionados en nuestro estudio de caso, las perspectivas teóricas y analíticas discutidas aportan una aproximación explicativa al tema de fondo de este trabajo, al señalarnos la evidencia de un problema mundial y transnacional, esto es, los cambios de percepción y orientación de los grupos de intereses chilenos en su relación con los Estados Unidos. Ante todo, destacamos el valor de la concepción estatista para analizar la interacción entre actores gubernamentales y privados en el marco de la atracción y legitimidad de las políticas estadounidenses mediante la difusión de los valores políticos, culturales y sociales que representa el llamado poder suave en la proyección internacional estadounidense. Una expresión concreta de esta política es la propia experiencia del Programa Fulbright, que ha contribuido a la formación de elites en Chile bajo la inspiración de valores educacionales y de la propagación de la cultura política de Estados Unidos.

Del mismo modo, la identificación y la sustentación conceptual de algunos resultados empíricos de la encuesta abordada por este estudio a partir del paradigma transnacional con las visiones más actuales sobre globalismo social y cultu- 
ral son evidentes. Así lo demuestran el alto reconocimiento y la aprobación de los entrevistados a los aportes que los medios de comunicación y el dominio del idioma inglés como lengua franca de la globalización entregan a las elites locales para conectarse e interactuar en el mundo actual.

El valor de la concepción estatista forma parte del análisis de la interacción entre actores gubernamentales y privados.

En el campo de la interacción entre variables internas y sistémicas de la política exterior y, específicamente en lo que dice relación con la explicación de percepciones de los actores seleccionados en este trabajo, los enfoques sobre estrategias de desarrollo y las perspectivas "trans-sociales" y "trans-gubernamentales" constituyen argumentos teóricos y analíticos sugerentes en los intentos de explicar y justificar las opciones de política exterior de nuestros encuestados. Las apreciaciones de los entrevistados en nuestro estudio confirman la alta valoración y prioridades que generan los acuerdos comerciales, particularmente con Estados Unidos, en términos de crecimiento y éxito de la estrategia económica chilena. Asimismo, estas percepciones se complementan con las expectativas de aumentar los vínculos transnacionales entre las economías y las sociedades en un plano de interdependencia creciente. De este modo, son elocuentes las coincidencias de opciones de las elites chilenas en la formulación y aplicación de una estrategia de inserción internacional, que apunta prioritariamente al desarrollo nacional, conjuntamente con el reconocimiento en la formación de vínculos y redes transnacionales entre agentes públicos y privados que interactúan regularmente en el ámbito de la política bilateral y mundial. 